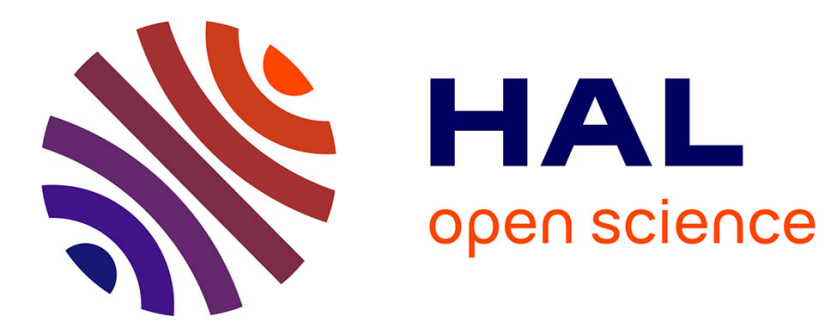

\title{
Les enjeux de la numérisation et de l'ouverture d'archives : le point de vue des professionnels
}

Karine Roudaut, Annabelle Boutet

\section{To cite this version:}

Karine Roudaut, Annabelle Boutet. Les enjeux de la numérisation et de l'ouverture d'archives : le point de vue des professionnels. Terminal. Technologie de l'information, culture \& société, 2012, 110, pp.27-37. 10.4000/terminal.1220 . hal-02018236

\section{HAL Id: hal-02018236 \\ https://imt-atlantique.hal.science/hal-02018236}

Submitted on 2 Sep 2019

HAL is a multi-disciplinary open access archive for the deposit and dissemination of scientific research documents, whether they are published or not. The documents may come from teaching and research institutions in France or abroad, or from public or private research centers.
L'archive ouverte pluridisciplinaire HAL, est destinée au dépôt et à la diffusion de documents scientifiques de niveau recherche, publiés ou non, émanant des établissements d'enseignement et de recherche français ou étrangers, des laboratoires publics ou privés. 


\title{
Les enjeux de la numérisation et de l'ouverture d'archives : le point de vue des professionnels
}

\author{
L'exemple de la production et la diffusion \\ d'un patrimoine local.
}

\author{
Karine Roudaut*, Annabelle Boutet**
}

$\mathrm{L}$ es technologies de l'information et de la communication (sites internet, fora de discussion, wikis, communautés de pratique en ligne, etc.) ont engendré le développement d'outils et de services, de modes de production, de création et de circulation de la connaissance, favorisant des formes de travail collaboratif à distance, l'émergence de collectifs ou encore le partage de répertoires communs. Ces nouvelles situations de travail sont porteuses de mutations socio-organisationnelles comme des formes nouvelles de « knowledge management " (gestion des connaissances) au sein des entreprises, l'engagement dans des communautés de pratiques ${ }^{2}$, le déplacement et le dépassement des frontières organisationnelles traditionnelles.

C'est dans ce contexte de développement des TIC que les organisations publiques - qui ont pour habitude de traiter les informations publiques qu'elles détiennent ou produisent comme des biens enclos (privatisés et mis à disposition de groupes fermés d'usagers) - mettent certains de leurs fonds à disposition du plus grand nombre sur Internet. Cette ouverture se traduit d'abord par la mise en ligne de contenus et induit ensuite la possibilité donnée aux

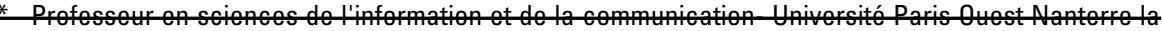

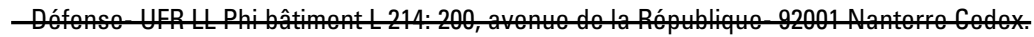
** Michel.arnaudparis10.fr.Tél.:06-8573-4057.

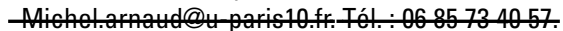

1. Les développements exposés dans cet article sont issus des réflexions menées à partir d'un projet de recherche porté par Annabelle Boutet et qui s'intitulait BICOOP, des Biens publics aux biens communs, Collaboration, Organisations et Pratiques. (www.marsouin.org/spip.php?article197)

2. Cette question de l'engagement dans des collectifs de production ouverte de connaissance est notam ment poursuivie dans les travaux de Nicolas Jullien et Karine Roudaut dans le cadre d'un projet de recherche ANR CONTINT intitulé CCCP-Prosodie (http://cccp-prosodie.org).
} 
« publics » de participer à leur enrichissement. Ce mouvement d'ouverture des biens publics informationnels au plus grand nombre pose la question générale de savoir comment les professionnels de ces organisations abordent, dans leurs pratiques et leurs représentations, les changements liés à l'introduction des outils et méthodes qui sous-tendent la société de l'information. Ces changements, introduits dans les situations d'activités, impliquent vraisemblablement des transformations dans la production des contenus, les méthodes de travail, la redéfinition des liens sociaux entre les acteurs concernés, tant les professionnels que leurs publics.

Les biens publics informationnels sont définis comme des biens ayant vocation à diffuser ou transmettre de la connaissance (des contenus). Ils sont publics du fait de leur relation forte à la collectivité, ce qui les rend, d'un point de vue naïf mais courant, non susceptibles d'être sujet à une appropriation privée à des fins marchandes. Ce mouvement d'ouverture des contenus comprend donc deux aspects : une mise à disposition des contenus dits publics (ou d'intérêt général) au plus grand nombre, et l'engagement des acteurs concernés dans des démarches collaboratives d'enrichissement de ces contenus. Définissant une autre manière d'exercer les missions publiques en permettant, notamment une plus grande participation citoyenne, cette tendance supporte des enjeux, sociaux, économiques, politiques, juridiques.

Selon nous, les actions portées par le développement des techniques de numérisation dans les métiers de la documentation et de l'archivage publics peuvent être abordées dans une vision de ce qu'est aujourd'hui l'exercice de la démocratie en France, avec les droits, pouvoirs et compétences accordés aux citoyens. En effet, l'administration descendante est chahutée par deux courants : le libéralisme défenseur de la dérégulation en faveur du partenariat ; l'émergence d'une culture citoyenne prônant les bienfaits de l'intelligence collective et de la participation. Ces actions nourrissent des discours autour de la démocratisation par la mise à disposition des citoyens des données publiques ; à ce titre, l'e-administration fait figure de laboratoire d'une standardisation des relations entre les institutions publiques et les citoyens (ou administrés) pour des besoins élémentaires identifiés.

Pour résumer la thèse que nous développerons dans cet article, nous étudierons comment les projets de numérisation d'archives municipales et culturelles [re-]positionnent la notion de démocratisation en suivant trois axes que nous qualifierons de civique, de professionnel et de marchand : $(1$ : civique $)$ la question de mise à disposition pour tous des données dites publiques ; $(2$ : professionnel) les dispositifs d'appropriation de ces données publiques déployés par les professionnels et les tensions qu'ils génèrent avec d'autres aspects de ces métiers ; ( 3 : marchand) la dimension économique générée par une situation de plus en plus concurrentielle dans laquelle se trouvent les ins- 
titutions publiques qui doivent multiplier les actions pour attirer ou générer des ressources, ceci passant par la captation de « publics » nouveaux ou plus nombreux et la preuve de leur utilité publique et politique.

Nous décrirons comment, chacune à leur niveau, ces dimensions se heurtent ou viennent heurter des situations, des dispositions, et des dispositifs professionnels et organisationnels ; mais aussi comment elles se télescopent les unes les autres. C'est pourquoi nous chercherons à expliquer les enjeux, les freins et les facilitations à la mise à disposition du plus grand nombre par des organisations semi-publiques et publiques.

L'enquête s'est déroulée de novembre à décembre 2008 auprès d'acteurs des Archives Municipales et Communautaires de la Ville de Brest, de l'association de la Cinémathèque de Bretagne, et d'associations brestoises telles que la Caisse à clous, la Maloïne (Rue de Saint-Malo) et Brest-à-l'œuvre, tous portant des projets de collecte de mémoire photographiques, sonores, iconographiques ou audiovisuelles sur le patrimoine culturel de Brest : mémoire ouvrière et métiers du port, mémoire vivante du quartier de Recouvrance (à Brest), mémoire de Brest comme objet esthétique et poétique.

Dans le cadre de ce projet, la méthode de collecte de données adoptée est de type qualitatif à base d'entretiens semi-directifs. Ils ont une durée de cinquante minutes à deux heures. Nous avons rencontré quinze personnes mais les résultats que nous présentons sont produits à partir des discours de trois « praticiens » des archives municipales et communautaires et de trois praticiens de la Cinémathèque de Bretagne. Les entretiens ont permis de dégager les significations des acteurs eux-mêmes à propos de leurs pratiques, de leurs expériences et du sens qu'ils donnent à leurs actions. Au début de ce projet, nous avons posé le postulat que la diffusion des innovations informationnelles et des pratiques qui leur étaient liées relevait d'un processus sociotechnique de type réticulaire, que cette diffusion pouvait dépasser le cadre organisationnel du modèle bureaucratique et impliquait des instances et des individus au-delà des frontières organisationnelles. C'est pourquoi, nous avons adopté l'option de poser le « projet » comme cadre d'intervention et non l'organisation instituée. À ce titre, les dimensions, institutionnelles et individuelles qui ont été investiguées sont les suivantes : l'activité, le travail effectué par l'acteur; le lien de l'acteur avec le projet (ici la numérisation et l'ouverture des données) ; l'engagement dans le projet en question; l'état des lieux et les perspectives du projet; le parcours professionnel.

Nous présenterons d'abord le contexte sociopolitique qui cadre cette étude, celui de la diffusion des données publiques et la numérisation du patrimoine, puis les enjeux de la numérisation et de l'ouverture des données (section 2). Enfin, suivra une analyse des effets observés sur les terrains étudiés (section 3). 


\section{Les enjeux de la diffusion et de la numérisation des données publiques : de la conservation à la patrimonialisation des fonds documentaires locaux.}

Nous expliquerons dans ce paragraphe comment les décisions et directions politiques en matière de conservation des données publiques conduisent progressivement les acteurs concernés à des démarches de patrimonialisation.

En effet, cette politique d'obligation de mise à disposition des données publiques et de diffusion contient plusieurs enjeux : économiques, relatifs à une mise à disposition de ces données gratuites et/ou à leur marchandisation ; organisationnels et professionnels, en lien avec un élargissement des compétences administratives (organisation) et/ou une transformation du métier d'archiviste ; politiques, autour de la définition des « données essentielles ", des modes de définition, des acteurs qui les définissent (experts et/ou citoyennetés).

Les enjeux économiques portent essentiellement sur le choix d'un modèle économique permettant l'accessibilité au plus grand nombre et en même temps la viabilité des dispositifs organisationnels et techniques qui les soutiennent. Les enjeux sociaux portent quant à eux sur les mutations des dispositifs organisationnels et des métiers concernés en termes notamment de missions, de compétences. Le troisième enjeu d'ordre politique est celui de la définition des données dites essentielles, autrement dit les biens qui font l'objet d'un traitement en vue de l'archivage.

\section{De l'obligation de diffusion des données publiques...}

Le rapport Mandelkern ${ }^{3}$ sur la diffusion des données publiques éclaire le contexte sociétal dans lequel s'inscrit notre question. Il pose les bases de tout ce qui suit en matière de diffusion des données publiques par voie numérique. Deux points sont présentés : complétant les droits ouverts aux citoyens en termes «d'accès aux documents administratifs » en 1978 par les lois « informatique et libertés », il défend l'obligation de mettre à disposition les données publiques numérisées, en ajoutant une dimension collective là où on avait jusqu'à présent un accès restreint et individuel. En outre, on attend de l'ouverture aux réseaux numériques mondiaux une incitation à de nouveaux usages, rendus possibles par l'accroissement de la visibilité des contenus français ${ }^{4}$.

Une première conséquence est de revoir la manière dont les biens informationnels sont mis à disposition, puisqu'il ne s'agit plus simplement de

3. Mandelkern D. (1999), Diffusion des données publiques et révolution numérique, rapport rédigé à la suite des travaux de l'Atelier présidé par Monsieur Mandelkern au Commissariat au Plan, Paris, La Documentation Française, $124 \mathrm{p}$.

4. Mandelkern D., 1999, p. 4. 
faciliter l'accès à des requêtes individuelles mais de déployer des dispositifs sociotechniques pour une diffusion au plus grand nombre. La seconde conséquence immédiate est de redéfinir ce qui est proposé à la diffusion, autrement dit les contenus. Comme le souligne le rapport, «l'obligation faite aux services publics de permettre, dans certaines conditions, l'accès aux informations qu'ils détiennent se transforme en une obligation de les diffuser », ce qui nécessite aussi d'assurer « la mise à jour permanente de leur contenu $»^{5}$.

Deux contraintes sont mises en exergue : celui de l'interactivité et celui des modèles économiques pris entre les principes de la gratuité et ceux de la marchandisation. La demande d'interactivité conduit à trois types de conséquences : elle repose sur un nouveau travail de « bornage » des compétences administratives ; elle remet en cause l'organisation interne du diffuseur public ; elle pousse à l'extension de la notion de « données essentielles » 6 .

\section{...à la patrimonialisation des contenus archivés}

Si la numérisation soutient l'obligation faite de diffuser les données publiques, elle engendre un effet non prescrit d'élargissement et d'enrichissement des contenus qui, à son tour, produit une injonction à fournir de nouveaux produits. Ainsi, elle contribue à transformer la perception que les professionnels des archives ont ou pourraient avoir de leur rôle et de leurs méthodes de travail, particulièrement pour répondre à la transformation d'une demande d'accès individuelle vers une diffusion de masse. À ce titre, les professionnels des archives ne seraient plus uniquement des conservateurs de biens publics mais des fournisseurs de biens culturels et patrimoniaux.

En effet, jusqu'à présent, en ce qui concerne les professionnels auprès desquels nous avons investigué, la diffusion en réel se faisait, soit par l'intermédiaire de requêtes individuelles sur place, soit grâce à l'organisation d'expositions, soit par le biais de contributions à des expositions (par le prêt de documents d'archives) organisés par des partenaires culturels, des événements comme «Les journées du Patrimoine » ou des rencontres autour d'archives audiovisuelles, etc. Aujourd'hui, les perspectives de diffusion devant un public élargi, ainsi que les enjeux de la concurrence, qui obligent à plus d'efficacité, incitent les professionnels des archives à enrichir leurs fonds, non plus simplement pour répondre à un souci d'historicité mais à des fins culturelles. Par ce processus, les fonds archivés peuvent devenir des biens patrimoniaux utilisés dans la promotion des collectivités locales. Car un des

5. Ibid. p.5.

6. Mandelkern D., 1999 : 33-34. «Les données essentielles seraient alors définies comme les données publiques dont la mise à disposition est une condition indispensable à l'exercice des droits du citoyen, ainsi que de ceux des étrangers résidants sur notre sol. Le critère de la finalité de l'usage commande une présentation des données essentielles sous une forme facilement et universellement accessible ". Voir Mandelkern D., 1999 : 95). La mise à disposition des données essentielles ne peut pas donner lieu à rémunération. 
principaux critères de sélection des contenus détenus par les archives locales est la recherche de la proximité et notamment de la proximité géographique. En d'autres termes, on trouve généralement dans les archives des documents se rapportant à des personnalités, lieux, événements locaux et qui sont définis par leur appartenance à un territoire.

Rapportés aux terrains que nous avons étudiés, les enjeux sont : ceux de la démocratisation de l'accès aux données et de leur appropriation par tous, ceux de la concurrence pour la conquête de nouvelles ressources et de nouveaux publics, ce qui pose la question de leur visibilité et de leur survie.

\section{Les effets}

Les projets de numérisation et d'ouverture des données publiques sont vécus par nos interlocuteurs comme des situations contraignantes associées à une vision techno-centrée plutôt déterministe avec l'obligation de suivre un mouvement irréversible de rationalisation vers toujours plus d'efficacité et d'accélération des temporalités. Pour certains, ils sont imposés par la loi ; pour d'autres, ils relèvent de choix organisationnels ou institutionnels. Loin d'être neutre, la technique, associée à un environnement social et politique de diffusion des données au plus grand nombre, cristallise l'expression d'une tension forte entre un principe citoyen, une logique industrielle, et un modèle marchand.

\section{La mise à disposition des données : un enjeu pour la citoyenneté}

Les notions de démocratisation du patrimoine, de diffusion collective et de mise à disposition du plus grand nombre caractérisent une définition de bien commun, entendu comme un bien public dont chaque citoyen peut disposer. Dans les entretiens réalisés, la mise à disposition et la diffusion «pour tous », " au plus grand nombre », prennent la forme de valeurs supérieures érigées en « devoir ». Nous avons en ce sens identifié l'importance que représente traditionnellement l' " obligation de la preuve » dans la mission de conservation par les archives qui vient s'enrichir aujourd'hui de la responsabilité de conserver des traces " pour les générations futures».

Pour les acteurs interviewés des archives municipales, " être un service public » ouvert aux citoyens passe notamment par une culture de la gratuité. Pour autant, si la démocratisation recouvre une logique de justification de service public citoyen pour les données dites essentielles (Mandelkern, 1999 : 95), la gratuité de tout autre type de données peut être débattue, elle relève d'un choix politique.

L'ambivalence de la numérisation a des effets que nous avons constatés et dont nous pouvons souligner l'interdépendance : la professionnalisation des publics ; la visibilité des agences d'archives ; la concurrence et la marchandisation. 


\section{La professionnalisation des publics}

La mise à disposition des données au plus grand nombre débouche dans les faits sur une professionnalisation des usagers et une spécification des demandes. En effet, les publics concernés ou intéressés par les archives sont, pour la plupart d'entre eux, des professionnels et des amateurs éclairés. Ce sont, par exemple, les généalogistes, les chercheurs amateurs ou les universitaires, les professionnels de l'audiovisuel. Les métiers des archives y voient une valorisation et une reconnaissance de leur activité portée par la technologie, ce qui renforce leur statut d'expert. L'élargissement du rayonnement et la visibilité des contenus détenus par ces archives permettent un gain de notoriété pour le travail effectué et les compétences développées par les archivistes. Nous retrouvons ici le « mythe Internet » de l'échange déterritorialisé et de la proximité hors les frontières, renforcé par l'idée d'appartenir à une même communauté de pratique autour d'un domaine (les archives), d'une identité et de méthodes. Les professionnels des archives s'ouvrent aussi vers d'autres pratiques d'échange de connaissances, de partage d'informations, de contenus et d'expériences ; en d'autres termes ils développent également de nouvelles formes collaboratives d'exercice de leurs activités, par l'utilisation des services numériques.

Ce processus de démocratisation et d'ouverture reste, dans l'ensemble, partiel car l'accès généralisé n'est pas atteint. Accéder à des contenus numérisés suppose, de fait, un prérequis minimal de familiarisation avec les Technologies de l'Information et de la Communication.

Ce constat justifie la professionnalisation des publics. Le processus de démocratisation que l'on voudrait vertueux produit des effets pervers sous formes de phénomènes d'exclusion ; il existe un hiatus entre des publics susceptibles d'être intéressés par les contenus proposés et des compétences techniques que leur accès impose.

\section{La visibilité des agences d'archives}

L'efficacité et la compétence professionnelle tendent vers le développement d'un mode de fonctionnement plus industriel en lien avec la conservation des données numériques ou dématérialisées. En effet, la conservation de ce type de données offre aux services des archives municipales une nouvelle place au sein de l'administration dans le traitement des questions relatives à l'e-administration et l'archivage électronique. Les professionnels sont maintenant consultés dès « la création même des documents » et n'interviennent plus simplement en « bout de la chaîne ». Nous nous autorisons à dire que les archives ne sont plus « la dernière porte au fond du couloir » mais qu' elles ont ouvert une nouvelle " fenêtre sur cour ». La numérisation contient un enjeu d'image et de valorisation indéniable 
d'un point de vue org a $\mathrm{n}$ i s a t i o $\mathrm{n} \mathrm{n}$ el.Le soutien financier apporté dans les projets de numérisation atteste que la préservation de la mémoire n'est plus seulement un enjeu de conservation, domaine réservé des organisations d'archives, mais un espace de luttes pour la définition d'une identité territoriale et la captation des fonds culturels qui la nourrissent.

\section{Concurrence et marchandisation}

L'existence d'un débat autour de la mise en œuvre d'un modèle marchand ressort également des analyses : les questions de la rentabilisation de la conservation des contenus et de leurs modes de diffusion n'ont jamais été aussi vives. L'accumulation des biens dits culturels, si possible rares, entre dans une logique de patrimonialisation. La question des coûts des projets de numérisation de certains fonds, des coûts des supports techniques nécessaires à cette activité, conduit, entre autre, à externaliser la numérisation auprès de cabinets professionnels de généalogistes, à des partenariats avec des entreprises privées et à la recherche de financements externes à la collectivité par le biais des appels d'offre ou de la vente d'images.

Les représentations qui sont associées à ces pratiques ne sont pas nécessairement perçues négativement, et ce malgré l'affirmation d'un principe citoyen. Au contraire, l'enquête souligne que ces pratiques peuvent être valorisées positivement et sont justifiées selon plusieurs registres : soit parce qu'elles représentent un moindre coût pour la collectivité, soit parce qu'elles permettent la viabilité économique de la structure, lorsque celle-ci ne relève pas entièrement du service public, mais doit assurer une part d'autofinancement.

Cette dimension de la concurrence des contenus et de leur marchandisation oriente même parfois les méthodes de travail des archives, particulièrement celles relevant du tri et de l'indexation. Ainsi le tri des biens culturels peut désormais engendrer le refus d'un dépôt justifié par le manque de place ou le coût de la conservation et du stockage. Les critères de l'intérêt et de l'utilité sont évoqués, ce qui pose la question du choix des contenus numérisés et de leur mise à disposition. Tous les points de vue recueillis convergent sur le fait que les documents numérisés et mis en ligne sont ceux qui sont le plus demandés.

Certes, ils invoquent la logique de la conservation pour éviter ou réduire les manipulations qui endommagent, à force de répétition, les documents, mais ils mettent également en avant l'utilité, entendue au sens de l'intérêt économique, de mettre en ligne des documents qui rencontrent trop peu de public. La prise en compte de cet argument, associé à une certaine idée d'appropriation du patrimoine, peut conduire des instances à retravailler $\mathrm{d}$ e $\mathrm{s}$ documents ou des objets, tels que des films.

Ainsi, les enjeux sont forts autour de deux courants : produire et ven- 
dre des films thématiques accessibles au plus grand nombre à partir des documents d'origine ou diffuser des documents bruts, tels qu'ils sont produits par les cinéastes amateurs. Ici, les sources de tensions sont liées au fait que l'on s'éloigne, d'une certaine manière, de la conservation comme cœur de métier des archives et de leur identité.

Fondamentalement l'enquête permet de montrer que ce n'est pas la diffusion au plus grand nombre via Internet qui pose problème ou pourrait freiner l'engagement vers de tels projets de numérisation et d'ouverture, mais les méthodes d'ouverture de ces données. À ce titre, les sources de tension sont liées à l'analyse ou à la mise à l'épreuve que les professionnels accordent à leurs interactions avec les publics et les données elles-mêmes. Ainsi, garder la maîtrise des données qui sont mises en ligne est une préoccupation forte de la part des professionnels qui s'estiment responsables de la qualité de ce qu'ils offrent.

Cette responsabilité est mise en balance face à la représentation selon laquelle Internet est un vaste espace peu normalisé et qui ouvre une incertitude quant à l'avenir des données publiées. En outre, la responsabilité des professionnels est justifiée par leur expertise et leurs compétences face à des publics dont les ressources en matière de compréhension des données mais aussi en matière de maîtrise des techniques sont questionnées ; les professionnels déplorent ainsi la difficulté qu'il y a d'avoir une démarche pédagogique ou d'accompagnement vers des publics qui ne sont plus accessibles directement mais au contraire mis à distance par la technique. Enfin, ils relèvent l'ambiguïté de la démarche de publicisation liée au fait que, d'une part, cette démarche a une vocation démocratique mais que, d'autre part, elle nécessite une certaine maîtrise technique pour une appropriation sociale du patrimoine ainsi valorisé.

\section{Conclusion}

La mise à disposition des données n'est jamais conçue comme une ouverture complète. Les freins identifiés sont de plusieurs types. Il existe une réelle tension face aux conséquences envisagées : tension entre d'une part, l'impression que les missions des archivistes sont réévaluées et d'autre part, l'absence de contrôle des trajectoires des données publiées conduisent à un pillage ou à une dénaturation de ce même travail. Il y a, en effet, tout un travail de conservation, d'indexation qui, s'il est invisible pour l'œil profane, n'en est pas moins fondamental pour que les archives soient accessibles. Nous retrouvons ici les questionnements relatifs à l'invisibilité du travail et la reconnaissance de l'activité professionnelle. Comme le montraient déjà Susan Leigh Star et Anselm Strauss 7 , "d'un côté, la visibilité peut signifier

7. Susan Leigh Star \& Anselm Strauss (1999), "Layers of Silence, Arenas of Voice : The Ecology of Visible and Invisible Work ", Computer Supported Cooperative Work 8: 9-30. 
la légitimité »; de l'autre, elle " peut créer une sorte de réification du travail, des possibilités de surveillance » ou de contrôle du travail effectué, " ou encore venir augmenter les charges de travail et la communication ». Elle peut aussi être source d'évaluation, dès lors qu'elle confronte les acteurs aux traces de leur activité8.

Le deuxième groupe d'arguments est d'ordre économique. Certains professionnels s'opposent à l'ouverture des données par crainte d'un détournement commercial, par des éditeurs privés, des données publiques, quand les coûts de gestion incombent aux archives. Il en va également de la viabilité économique de structures à économie mixte (ou semi-publiques), dans un contexte global peu enclin à élargir les ressources et les aides. Cette tension des intérêts est adossée à l'affirmation d'une revendication forte de service public. S'y ajoutent des arguments relatifs à l'absence de dispositifs techniques fiables ou de leur coût.

Enfin, les risques d'un détournement des contenus et de non respect des droits liés aux œuvres et aux auteurs sont récurrents dans ce que génère « le travail de la visibilité ». Dans les discours recueillis, si la modification ou la réutilisation des images est perçue comme légitime, elle ne l'est que tant qu'elle constitue une forme d'appropriation d'un patrimoine avec l'envie de le partager (en le rediffusant). Elle peut alors faire figure de réappropriation. Envisagée de la sorte, elle témoigne d'une attitude plutôt confiante mais sous certaines conditions d'usage : un contexte limité essentiellement au cercle des « proches », réunis dans une communauté de pratique ou au moins d'intérêts, et sans la perspective d'un usage marchand des contenus.

Pour conclure, il est important de souligner que les freins et les facilitations relèvent des mêmes dimensions d'usage. L'exemple le plus net est celui lié à la dimension du travail visible : l'espace virtuel étant aussi un moyen de compenser l'absence de lieu d'exposition et de diffusion propre à ces archives. Ce qui pointe donc une ambivalence sur ce qu'apporte la diffusion des contenus à l'activité des professionnels : entre d'un côté une certaine visibilité, la reconnaissance et la légitimité de leur travail, d'un autre côté l'hétérogénéité et la coopération, mais aussi la concurrence, également du savoir des amateurs, ce qui constitue un des enjeux notamment présents dans les collectifs de production de connaissance ouverte, dont le projet de l'encyclopédie Wikipédia est un exemple emblématique.

8. Béatrice Cahour, Christian Licoppe (2010), "Confrontation aux traces de son activité. Compréhension, développement et régulation de l'agir dans un monde de plus en plus réflexif ", Revue d'anthropologie des connaissances, 2, 243-253. 


\section{RÉFÉRENCES}

CAHOUR B., LICOPPE C. (2010), "Confrontation aux traces de son activité. Compréhension, développement et régulation de l'agir dans un monde de plus en plus réflexif ", Revue d'an thropologie des connaissances, 2, 243-253.

MANDELKERN D. (1999), " Diffusion des données publiques et révolution numérique ".

Rapport rédigé à la suite des travaux de l'Atelier présidé par D. Mandelkern au Commissariat au Plan, Paris, La Documentation Française, 124p.

STAR S. L., STRAUSS A. (1999), Layers of Silence, Arenas of Voice: The Ecology of Visible and Invisible Work, Computer Supported Cooperative Work 8: 9-30. 